\title{
Capturing respiratory syncytial virus season in Belgium using the influenza severe acute respiratory infection surveillance network, season 2018/19
}

Lorenzo Subissi ${ }^{1,2}$, Nathalie Bossuyt ${ }^{3}$, Marijke Reynders ${ }^{4}$, Michèle Gérard ${ }^{5}$, Nicolas Dauby ${ }^{5,6}$, Marc Bourgeois ${ }^{7}$, Bénédicte

Delaere7, Sophie Quoilin ${ }^{3}$, Steven Van Gucht ${ }^{1}$, Isabelle Thomas ${ }^{1}$, Cyril Barbezange ${ }^{1}$

1. National Influenza Centre, Sciensano, Brussels, Belgium

2. European Public Health Microbiology Training Programme (EUPHEM), European Centre for Disease Prevention and Control, Stockholm, Sweden

3. Epidemiology of Infectious Diseases, Sciensano, Brussels, Belgium

4. Department of Laboratory Medicine, Medical Microbiology, Algemeen Ziekenhuis Sint-Jan, Brugge-Oostende AV, Belgium

5. Centre Hospitalier Universitaire St-Pierre, Brussels, Belgium

6. Centre for Environmental Health and Occupational Health, School of Public Health, Université Libre de Bruxelles (ULB), Brussels, Belgium

7. Centre Hospitalier Universitaire UCL Namur, Ysoir, Belgium

Correspondence: Cyril Barbezange (cyril.barbezange@sciensano.be)

Subissi Lorenzo, Bossuyt Nathalie, Reynders Marijke, Gérard Michèle, Dauby Nicolas, Bourgeois Marc, Delaere Bénédicte, Quoilin Sophie, Van Gucht Steven , Thomas Isabelle, Barbezange Cyril. Capturing respiratory syncytial virus season in Belgium using the influenza severe acute respiratory infection surveillance network, season 2018/19. Euro Surveill. 2020;25(39):pii=1900627. https://doi.org/10.2807/1560-7917.ES.2020.25.39.1900627

Background: Respiratory syncytial virus (RSV) is a common cause of severe respiratory illness in young children ( $<5$ years old) and older adults ( $\geq 65$ years old) leading the World Health Organization (WHO) to recommend the implementation of a dedicated surveillance in countries. Aim: We tested the capacity of the severe acute respiratory infection (SARI) hospital network to contribute to RSV surveillance in Belgium. Methods: During the 2018/19 influenza season, we started the SARI surveillance for influenza in Belgium in week 40, earlier than in the past, to follow RSV activity, which usually precedes influenza virus circulation. While the WHO SARI case definition for influenza normally used by the SARI hospital network was employed, flexibility over the fever criterion was allowed, so patients without fever but meeting the other case definition criteria could be included in the surveillance. Results: Between weeks 402018 and 2 2019, we received 508 samples from SARI patients. We found an overall RSV detection rate of $62.4 \%$ (317/508), with rates varying depending on the age group: $77.6 \%$ inchildren aged $<5$ years $(253 / 326)$ and $34.4 \%$ in adults aged $\geq 65$ years $(44 / 128)$. Over $90 \%$ of the RSV-positive samples also positive for another tested respiratory virus (80/85) were from children aged<5years. Differences were also noted between age groups for symptoms, comorbidities and complications. Conclusion: With only marginal modifications in the case definition and the period of surveillance, the Belgian SARI network would be able to substantially contribute to RSV surveillance and burden evaluation in children and older adults, the two groups of particular interest for WHO.

\section{Introduction}

Respiratory syncytial virus (RSV) is an important cause of acute lower respiratory infections in children [1]. Severe forms of disease caused by RSV, including pneumonia, can lead to hospitalisation resulting in several thousand deaths per year worldwide in hospitalised children under the age of 5 years [2]. RSV is also a major cause of severe acute respiratory infections (SARI) in older adults ( $\geq 65$ years old) [3-5]. Currently, in Europe, palivizumab is the only commercially-available antiviral designed against RSV infection, and specifically for children [6]. Vaccines are in development and are expected to become available in the coming years [7-9]. To help decision-makers and to evaluate the impact of future vaccination, a dedicated surveillance is necessary. This surveillance might shed light on several aspects of RSV epidemiology (e.g. seasonality, atrisk age groups, complications) in particular why/how certain parameters vary between different areas of the world [10].

In 2016, the World Health Organization (WHO) launched a pilot study to evaluate the feasibility of using the longstanding worldwide influenza surveillance network (Global Influenza Surveillance and Response System) for a sustainable RSV surveillance [10]. The attempt to implement such a surveillance aimed at better understanding the virus, its circulation patterns and the disease it causes in the different age groups to eventually refine case definitions [10]. In addition, this study tried to look into the health burden of RSV in order to identify at-risk groups who would most benefit from 


\section{FIGURE 1}

Flowchart for the inclusion of SARI patients in the RSV pilot study, Belgium, October 2018-January 2019 ( $n=508$ included patients) ${ }^{\mathrm{a}}$

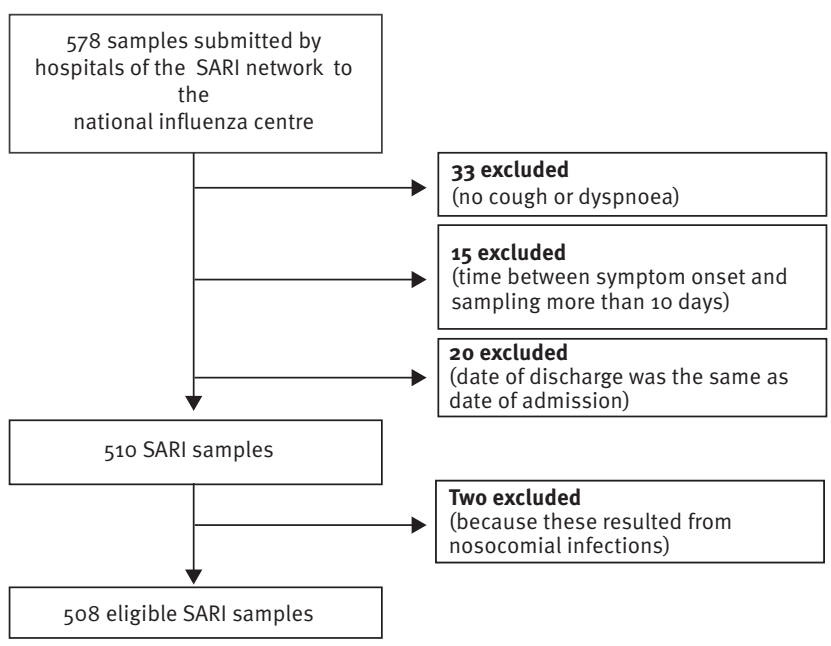

RSV: respiratory syncytial virus; SARI: severe acute respiratory infection.

a Only three of the six hospitals in the Belgian SARI surveillance network, one in each administrative region (Flanders, Brussels Capital, Wallonia), took part in this pilot study.

vaccination [10]. The case definition recommended for countries using a hospital-based surveillance strategy was an extended WHO SARI case definition for patients aged $\geq 6$ months. For those aged $<6$ months, the case definition should also include apnoea or sepsis [10]. Moreover, the sampling strategy recommended ca 1,000 samples per year per country, distributed among the following age groups: 16 months, 6 months to 4 years, 5 to 64 years, and $\geq 65$ years [10].

In Belgium, the national influenza centre recognised by WHO obtains influenza surveillance data through two networks. The first consists of general practitioners and concerns influenza-like illness (ILI) surveillance (from week 40 to week 20 of the following year; i.e. influenza season surveillance period); the second is constituted by six hospitals that monitor SARI (from the last week of December or first/second week of January to the third/last week of April, depending on when influenza virus circulation is detected by the ILI network; i.e. the influenza activity period). Historical data of the numbers of positive tests reported by all the Belgian hospital laboratories show that RSV circulation generally occurs sometime between October and January, and usually precedes or slightly overlaps the circulation of influenza viruses [11], like in neighbouring countries $[12,13]$.

Since these data do not provide enough information to assess the RSV burden, we decided to test the existing SARI network capability to contribute to RSV surveillance in Belgium.

\section{Methods}

Settings of the Belgian pilot study

During the 2018/19 influenza season (week 402018 to week 20 2019), three of the six hospitals of the SARI network, one in each administrative region (Flanders, Brussels-Capital, Wallonia), volunteered to start the SARI surveillance as early as week 40 2018, instead of at the end of December or beginning of January. Our usual SARI case definition, based on the WHO SARI case definition, was used: acute respiratory infection with fever $\geq 38^{\circ} \mathrm{C}$ (or history of fever reported by the patient) and cough or dyspnoea, with onset of symptoms within the past 10 days, and requiring hospitalisation (minimum overnight). However, in order to align with one of the case definitions proposed in WHO's RSV strategy document $[14,15]$, the participating hospitals were recommended to be flexible over the fever criteria, meaning that patients without fever but meeting the other criteria could also be included.

Unless consent was refused, enrolment included all patients meeting the case definition. Beside sampling (nasopharyngeal swab or aspirate), SARI standardised questionnaires were used to collect data on age, sex, symptoms, antibiotic treatment, known comorbidities and follow-up during hospitalisation to evaluate the disease severity.

\section{Laboratory investigation}

Respiratory samples were analysed at the national influenza centre. Viral nucleic acids were extracted using BioMerieux's NucliSENS EasyMag (Brussels, Belgium). Routine in-house multiplex reverse transcription quantitative (RT-q)PCRs were used to detect the following respiratory virus targets: adenoviruses, bocavirus, coronaviruses (CoV-OC43, CoV-NL63 and CoV-229E separately), human metapneumoviruses, influenza virus types $A$ and $B$, parainfluenzavirus types 1, 2, 3 and 4 (separately), parechovirus, picornaviruses (rhinovirus and enterovirus genera) with specifically enterovirus D68, and RSV types A and B (adapted from original protocols by United States Centers for Disease Control and Prevention; LJ van Elden, University Medical Centre Utrecht, the Netherlands; P Overduin, the National Institute for Public Health and the Environment, the Netherlands; O Hungnes and K Bragstad, Institute of Public Health, Norway). Primer and probe sequences and RT-qPCR conditions are available upon request.

\section{Statistical analysis}

A two-tailed Fisher's exact test (two-by-two table) or a chi-squared test was performed for group proportion comparisons. The Mann-Whitney test was used to compare the distributions of the length of stay in hospital. Differences were considered as statistically significant for a $\mathrm{p}$ value $<0.05$.

\section{Ethical statement}

The SARI surveillance protocol was approved by the central Ethical Committee (reference AK/12-02-11/4111; 
TABLE 1

Characteristics of SARI patients according to RSV and other respiratory virus test results, Belgium, week 412018 -week 2 $2019(n=508)$

\begin{tabular}{|c|c|c|c|c|c|c|c|c|c|c|c|}
\hline \multirow{3}{*}{ Characteristic } & & \multicolumn{4}{|c|}{ RSV negative } & \multicolumn{4}{|c|}{ RSV positive } & \multirow{2}{*}{\multicolumn{2}{|c|}{ Total }} \\
\hline & & \multicolumn{2}{|c|}{$\begin{array}{l}\text { Negative for all } \\
\text { other respiratory } \\
\text { virus tested }\end{array}$} & \multicolumn{2}{|c|}{$\begin{array}{l}\text { Positive for another } \\
\text { respiratory virus } \\
\text { tested }\end{array}$} & \multicolumn{2}{|c|}{ RSV only } & \multicolumn{2}{|c|}{ RSV co-infection ${ }^{\mathrm{a}}$} & & \\
\hline & & Number & $\%$ & Number & $\%$ & Number & $\%$ & Number & $\%$ & Number & $\%$ \\
\hline \multicolumn{2}{|l|}{ Overall } & 82 & 100 & 109 & 100 & 232 & 100 & 85 & 100 & 508 & 100 \\
\hline \multirow{4}{*}{ Age group } & $<6 \mathrm{~m}$ & 6 & 7.3 & 24 & 22.0 & 117 & 50.4 & 42 & 49.4 & 189 & 37.2 \\
\hline & $6 m-4 y$ & 12 & 14.6 & 31 & 28.4 & 56 & 24.1 & 38 & 44.7 & 137 & 27.0 \\
\hline & $5-64 y$ & 22 & 26.8 & 12 & 11.0 & 17 & 7.3 & 3 & 3.5 & 54 & 10.6 \\
\hline & $\geq 65 y$ & 42 & 51.2 & 42 & 38.5 & 42 & 18.1 & 2 & 2.4 & 128 & 25.0 \\
\hline \multirow{3}{*}{ Symptom } & Fever & 65 & 79.3 & 96 & 88.1 & 211 & 90.9 & 82 & 96.5 & 454 & 89.4 \\
\hline & Cough & 66 & 80.5 & 96 & 88.1 & 213 & 91.8 & 75 & 88.2 & 450 & 88.6 \\
\hline & Dyspnoea & 60 & 73.2 & 66 & 60.6 & 126 & 54.3 & 44 & 51.8 & 296 & 58.3 \\
\hline \multirow{12}{*}{ Comorbidity } & No & 20 & 24.4 & 45 & 41.3 & 143 & 61.6 & 65 & 76.5 & 273 & 53.7 \\
\hline & Yes & 62 & 75.6 & 64 & 58.7 & 89 & 38.4 & 20 & 23.5 & 83 & 16.3 \\
\hline & Chr. respi. & 27 & 32.9 & 22 & 20.2 & 28 & 12.1 & 6 & 7.1 & 83 & 16.3 \\
\hline & Asthma & 5 & 6.1 & 7 & 6.4 & 10 & 4.3 & 3 & 3.5 & 25 & 4.9 \\
\hline & Chr. cardio. & 18 & 22.0 & 24 & 22.0 & 21 & 9.1 & 2 & 2.4 & 65 & 12.8 \\
\hline & Renal insuf. & 11 & 13.4 & 13 & 11.9 & 15 & 6.5 & 3 & 3.5 & 42 & 8.3 \\
\hline & Hep. insuf. & 4 & 4.9 & 6 & 5.5 & 4 & 1.7 & 0 & 0.0 & 14 & 2.8 \\
\hline & Obesity & 13 & 15.9 & 5 & 4.6 & 4 & \begin{tabular}{|l|}
1.7 \\
\end{tabular} & 1 & 1.2 & 23 & 4.5 \\
\hline & Diabetes & 8 & 9.8 & 12 & 11.0 & 8 & 3.5 & 2 & 2.4 & 30 & 5.9 \\
\hline & Immunodef. & 6 & 7.3 & 15 & 13.8 & 22 & 9.5 & 3 & 3.5 & 46 & 9.1 \\
\hline & Neuromusc. & 5 & 6.1 & 10 & 9.2 & 12 & 5.2 & 0 & 0.0 & 27 & $5 \cdot 3$ \\
\hline & Unknown & 2 & ND & 0 & ND & 0 & ND & 0 & ND & 2 & ND \\
\hline \multirow{4}{*}{ Antibiotics } & No & 26 & 31.7 & 36 & 33.0 & 120 & 51.7 & 55 & 64.7 & 237 & 46.7 \\
\hline & Yes & 53 & 64.6 & 69 & 63.3 & 103 & 44.4 & 30 & $35 \cdot 3$ & 255 & 50.2 \\
\hline & Unknown & 1 & 1.2 & 0 & 0.0 & 1 & 0.4 & 0 & 0.0 & 2 & 0.4 \\
\hline & Missing & 2 & ND & 4 & ND & 8 & ND & 0 & ND & 14 & ND \\
\hline \multirow{3}{*}{ Death } & All ages & 7 & 8.5 & 8 & 7.3 & 6 & 2.6 & 1 & 1.2 & 22 & $4 \cdot 3$ \\
\hline & $5-64 y$ & 1 & ND & 2 & ND & 0 & ND & 1 & ND & 4 & ND \\
\hline & $\geq 65 y$ & 6 & ND & 6 & ND & 6 & ND & 0 & ND & 18 & ND \\
\hline \multirow{6}{*}{ Complication ${ }^{\mathrm{b}}$} & No & 45 & 54.9 & 62 & 56.9 & 126 & $54 \cdot 3$ & 50 & 58.8 & 283 & 55.7 \\
\hline & Yes $^{c}$ & 37 & 45.1 & 47 & 43.1 & 106 & 45.7 & 35 & 41.2 & 225 & $44 \cdot 3$ \\
\hline & Pneumonia & 19 & ND & 26 & ND & 36 & ND & 11 & ND & 92 & ND \\
\hline & ICU & 15 & ND & 11 & ND & 21 & ND & 4 & ND & 51 & ND \\
\hline & ARDS & 5 & ND & 10 & ND & 14 & ND & 5 & ND & 34 & ND \\
\hline & Resp. assis. & 19 & ND & 24 & ND & 77 & ND & 25 & ND & 145 & ND \\
\hline \multirow{5}{*}{ Stay in hospital } & Median & 6 & NA & 4 & NA & 5 & NA & 4 & NA & 6 & NA \\
\hline & Min & 1 & NA & 1 & NA & 1 & NA & 1 & NA & 1 & NA \\
\hline & $25 \%$ perc. & 3.8 & NA & 3 & NA & 3 & NA & 3 & NA & 3 & NA \\
\hline & $75 \%$ perc. & 12 & NA & 10.5 & NA & 8 & NA & 6 & NA & 8 & NA \\
\hline & Max & 37 & NA & 104 & NA & 56 & NA & 14 & NA & 104 & NA \\
\hline
\end{tabular}

ARDS: acute respiratory distress syndrome; chr. cardio.: chronic cardiovascular disease; chr. respi.: chronic respiratory disease; hep. insuf.: hepatic insufficiency; ICU: intensive care unit; immunodef.: immunodeficiency; m: months; max: maximum; min: minimum; NA: not applicable; ND: not determined; neuromusc.: neuromuscular disease; perc.: percentile; renal insuf.: renal insufficiency; resp. assis.: requirement for respiratory assistance (invasive and non-invasive); RSV: respiratory syncytial virus; SARI: severe acute respiratory infection; y: years.

${ }^{a}$ Other respiratory viruses tested: adenoviruses, bocavirus, coronaviruses (CoV-OC43, CoV-NL63 and CoV-229E), human metapneumoviruses, influenza virus types $A$ and $B$, parainfluenzaviruses (types 1, 2, 3 and 4 ), parechovirus, picornaviruses of the rhinovirus and enterovirus genera, specific enterovirus D68.

${ }^{\mathrm{b}}$ Other than death.

c At least one of the following: admission to ICU, detection of pneumonia based on chest radiography, development of ARDS, requirement for respiratory assistance.

d In days.

Number of patients and percentage within laboratory result category are presented. 
Weekly number of samples testing positive for RSV (A) captured by the SARI pilot surveillance and (B) reported by the NRC $^{a}$, Belgium, week 412018 -week2 $2019(n=508)$

\section{A. SARI pilot surveillance}

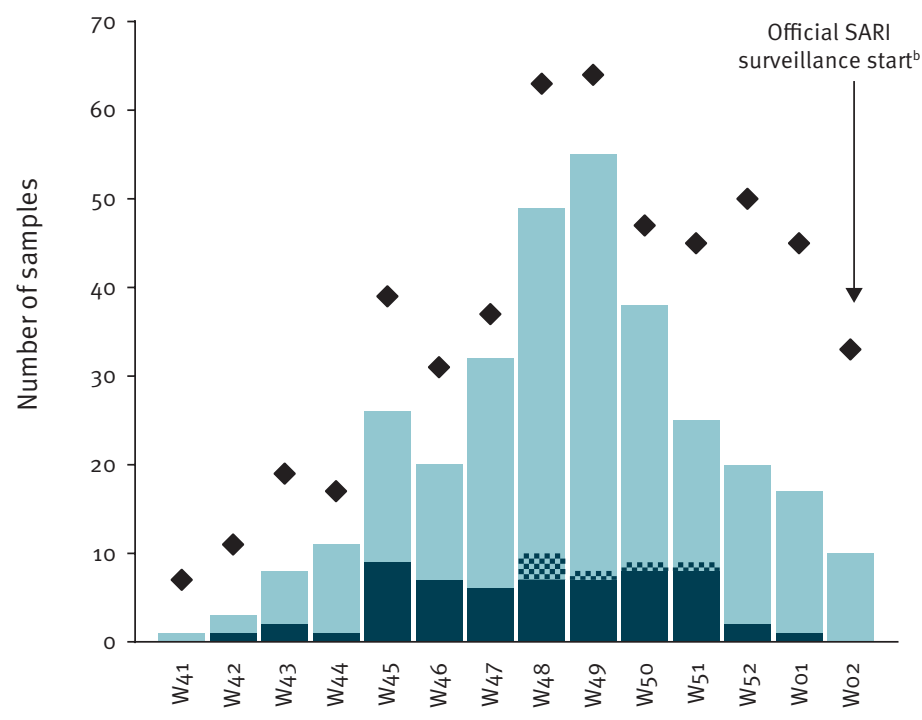

\section{B. National reference centre}

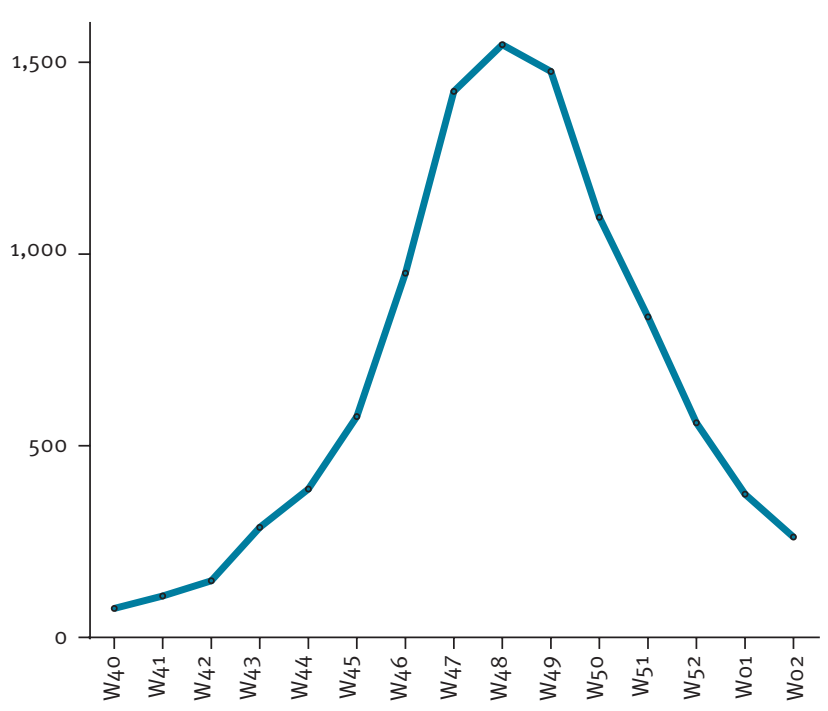

Week of sampling

$\begin{array}{ll}\text { RSV-B } & \text { RSV } \\ \text { RSV-A } & \text { RSV-A and RSV-B Samples tested }\end{array}$

NRC: national reference centre for respiratory pathogens; RSV: respiratory syncytial virus; SARI: severe acute respiratory infection.

a The distribution is based on the total number of RSV-positive tests reported to the NRC by the hospital laboratories in Belgium (including outpatients and inpatients, without any case definition; typing was not performed).

b The arrow indicates the start of the seasonal official SARI surveillance, which, in 2019, began in week 2 . Compared to the pilot surveillance, the official SARI surveillance involves three additional hospitals.

For the SARI surveillance (panel A), the weekly number of samples tested are represented by diamonds. The number of RSV-positive samples in the histograms are stacked, with different colours representing samples positive for RSV-A, RSV-B, or both RSV-A and RSV-B.

in 2011: Centre Hospitalier Universitaire St-Pierre, Brussels, Belgium; since 2014: Universitair Ziekenhuis Brussel, Brussels, Belgium) and the local ethical committees of the hospitals. The amendment for the pilot study was specifically approved by the central Ethical Committee and the local ethical committees of the participating hospitals. Informed consent was obtained from all participants or parents/guardians.

\section{Results}

Capturing the respiratory syncytial virus season through the SARI network

From week 402018 until week 2 2019, the week of the official start of the influenza SARI surveillance for the six hospitals, the national influenza centre received a total of 578 samples from the three hospitals participating in the study, of which 508 were eligible based on the adopted case definition (Figure 1). The median number of samples per week was 38 (interquartile range (IQR):18.5-37.8). The three sites each contributed to $36.0 \%$ (183/508), 39.2\% (199/508) and $24.8 \%$ $(126 / 508)$ of the total samples. There were slightly more samples from males $(55.1 \%, 280 / 508)$ than females. The WHO's recommended four age groups were covered, but the patients aged between 5 and 64 years were less represented (Table 1) and differences between sites were noted (data not shown). The weekly distribution of the number of RSV-positive samples among the SARI patients (Figure $2 \mathrm{~A}$ ) appeared to match the epidemiological curve reported by the national reference centre for respiratory pathogens (Figure 2B). Among the SARI patients, the peak of RSV infections was reached at week 49 with 55 RSV-positive samples (of 64 tested). Overall, during the study period, $62.4 \%(317 / 508)$ of the samples were positive for RSV, with RSV-B dominating during the $2018 / 19$ season.

Detection rates decreased with age (Table 1): $84.1 \%$ among infants aged $<6$ months (159/189), 68.6\% among 
Weekly RSV detection among SARI patients by age group, Belgium, week 41 2018-week2 2019

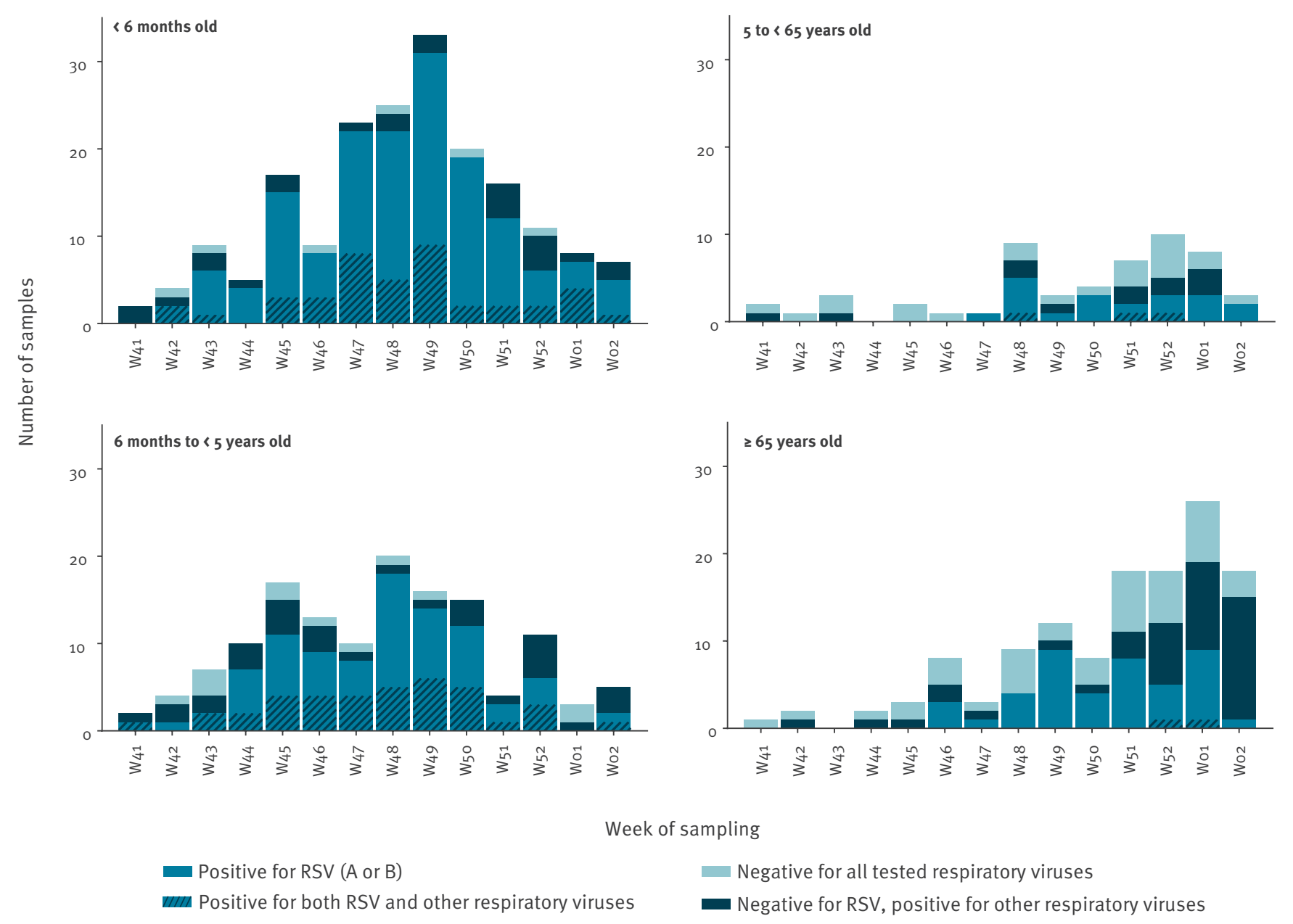

SARI: severe acute respiratory infection; RSV: respiratory syncytial virus; WHO: World Health Organization.

Age groups were defined as in the WHO's pilot study documents. In the histograms, numbers of samples per result categories, represented in different colours, are stacked (cumulative).

the 6-month-to-4-year-old children (94/137), $37.0 \%$ among the 5-to-64-year-old patients (20/54) and $34.4 \%$ among the older adults (44/128). The weekly distributions of the number of positive samples were similar for the two younger age groups, with a clear bell-shape curve starting as early as week 41 (Figure 3 ). On the other hand, for the 5-to-64- and $\geq 65$-year age groups, the first positive samples were detected only from week 47 and 46, respectively, and the curve resulting from the distribution did not present a clear peak.

Among the RSV-positive samples (Table 1), $26.8 \%$ (85/317) were also positive for at least one other respiratory virus tested, while this proportion was $57.1 \%$ among the RSV-negative samples (109/191). There was very good evidence of differences between age groups in the proportion of positivity (Table 1; chisquared test $\mathrm{p}<0.001)$. Among children aged $<5$ years, $53.1 \%(173 / 326)$ tested positive only for RSV, $24.5 \%$ (80/326) tested positive for RSV and at least one other respiratory virus investigated, nearly $17 \%$ (55/326) tested positive for a respiratory virus other than RSV (Supplement Table S1), and less than 6\% (18/326) were negative for all tested respiratory viruses. In contrast, among patientsaged $\geq 5$ years, more than one third $(64 / 182)$ tested negative for all investigated respiratory viruses, $32 \%(59 / 182)$ tested positive for only RSV, nearly $30 \%(54 / 182)$ tested positive only for a respiratory virus other than RSV, and less than 3\% (5/182) tested positive for RSV and at least one other respiratory virus of the panel (Supplement Table S1).

\section{Clinical signs included in the SARI case definition and other symptoms}

The data for fever, cough and dyspnoea are presented in Table 2 and Supplement Table S1, and summarised in Figure $4 \mathrm{~A}$. Fever was more common among children aged $<5$ years than among patients aged $\geq 5$ years regardless of the RSV status (with fever, RSV-negative: $93.2 \%(68 / 73)$ for<5years old vs $78.8 \% \quad(93 / 118)$ for $\geq 5$ years old, Fisher exact test $p=0.008$; with fever, RSV-positive: $95.3 \%(241 / 253)$ for $<5$ years old vs $81.3 \%$ 
TABLE 2

Characteristics of SARI patients according to age group and by result category, Belgium, week $412018-$ week 22019 ( $\mathrm{n}=508)$

\begin{tabular}{|c|c|c|c|c|c|c|c|c|}
\hline \multirow{2}{*}{ Characteristic } & & & \multicolumn{2}{|c|}{ RSV negative } & \multicolumn{2}{|c|}{ RSV positive } & \multicolumn{2}{|c|}{ Total } \\
\hline & & & Number & $\%$ & Number & $\%$ & Number & $\%$ \\
\hline \multicolumn{3}{|l|}{ Overall } & 191 & 100 & 317 & 100 & 508 & 100 \\
\hline Age & \multicolumn{2}{|c|}{$\geq 5 y$} & 118 & 61.8 & 64 & 20.2 & 182 & 35.8 \\
\hline \multirow{8}{*}{ Symptoms } & \multirow[b]{2}{*}{ All ages } & Fever & 161 & 84.3 & 293 & 92.4 & 454 & 89.4 \\
\hline & & Cough & 162 & 84.8 & 288 & 90.9 & 450 & 88.6 \\
\hline & \multirow{3}{*}{$<5 y$} & Fever & 68 & 93.2 & 241 & 95.3 & 309 & 94.8 \\
\hline & & Cough & 67 & 91.8 & 233 & 92.1 & 300 & 92.0 \\
\hline & & Dyspnoea & 29 & 39.7 & 118 & 46.6 & 147 & 45.1 \\
\hline & \multirow{3}{*}{$\geq 5 y$} & Fever & 93 & 78.8 & 52 & 81.3 & 145 & 79.7 \\
\hline & & Cough & 95 & 80.5 & 55 & 85.9 & 150 & 82.4 \\
\hline & & Dyspnoea & 97 & 82.2 & 52 & 81.3 & 149 & 81.9 \\
\hline \multirow{5}{*}{ Comorbidity $^{\mathrm{a}}$} & All ages & Yes & 126 & 66.0 & 109 & 34.4 & 83 & 16.3 \\
\hline & \multirow{2}{*}{$<5 y$} & None & 56 & 76.7 & 205 & 81.0 & 261 & 80.1 \\
\hline & & Yes & 17 & 23.3 & 48 & 19.0 & 65 & 19.9 \\
\hline & \multirow{2}{*}{$\geq 5 y$} & None & 9 & 7.6 & 4 & 6.3 & 13 & 7.1 \\
\hline & & Yes & 109 & 92.4 & 60 & 93.8 & 169 & 92.9 \\
\hline \multirow{16}{*}{ Complications $^{\mathrm{b}}$} & & No & 107 & 56.0 & 176 & 55.5 & 283 & 55.7 \\
\hline & & Yesc & 84 & 44.0 & 141 & 44.5 & 225 & 44.3 \\
\hline & All arec & Pneumonia & 45 & ND & 47 & ND & 92 & ND \\
\hline & mil ases & ICU & 26 & ND & 25 & ND & 51 & ND \\
\hline & & ARDS & 15 & ND & 19 & ND & 34 & ND \\
\hline & & Resp. assis. & 43 & ND & 102 & ND & 145 & ND \\
\hline & & No & 46 & 63.0 & 152 & 60.1 & 198 & 60.7 \\
\hline & & Yes $^{c}$ & 27 & 37.0 & 101 & 39.9 & 128 & 39.3 \\
\hline & & Pneumonia & 8 & ND & 25 & ND & 33 & ND \\
\hline & & Resp. assis. & 21 & ND & 82 & ND & 103 & ND \\
\hline & & No & 61 & 51.7 & 24 & 37.5 & 85 & 46.7 \\
\hline & & Yes $^{c}$ & 57 & 48.3 & 40 & 62.5 & 97 & 53.3 \\
\hline & & Pneumonia & 37 & ND & 22 & ND & 59 & ND \\
\hline & $25 y$ & ICU & 26 & ND & 19 & ND & 45 & ND \\
\hline & & ARDS & 12 & ND & 7 & ND & 19 & ND \\
\hline & & Resp. assis. & 22 & ND & 20 & ND & 42 & ND \\
\hline & & & 15 & 7.9 & 7 & 2.2 & 22 & 4.3 \\
\hline Death & & & 3 & 8.8 & 1 & 5.0 & 4 & 7.4 \\
\hline & & & 12 & 14.3 & 6 & 13.6 & 18 & 14.1 \\
\hline & & Median & 5 & NA & 5 & NA & 5 & NA \\
\hline & & Min & 1 & NA & 1 & NA & 1 & NA \\
\hline & All ages & $25 \%$ percentile & 3 & NA & 3 & NA & 3 & NA \\
\hline & & $75 \%$ percentile & 11 & NA & 7 & NA & 8 & NA \\
\hline & & $\operatorname{Max}$ & 104 & NA & 56 & NA & 104 & NA \\
\hline & & Median & 3 & NA & 4 & NA & 4 & NA \\
\hline & & $\operatorname{Min}$ & 1 & NA & 1 & NA & 1 & NA \\
\hline Length of stay ${ }^{d}$ & $<5 y$ & $25 \%$ percentile & 2 & NA & 3 & NA & 2 & NA \\
\hline & & $75 \%$ percentile & 4 & NA & 6 & NA & 5 & NA \\
\hline & & $\operatorname{Max}$ & 19 & NA & 32 & NA & 32 & NA \\
\hline & & Median & 9 & NA & 8.5 & NA & 9 & NA \\
\hline & & Min & 1 & NA & 1 & NA & 1 & NA \\
\hline & $\geq 5 y$ & $25 \%$ percentile & 5 & NA & 5 & NA & 5 & NA \\
\hline & & $75 \%$ percentile & 16 & NA & 14.8 & NA & 15.3 & NA \\
\hline & & $\operatorname{Max}$ & 104 & NA & 56 & NA & 104 & NA \\
\hline
\end{tabular}

ARDS: acute respiratory distress syndrome; ICU: intensive care unit; max: maximum; min: minimum; NA: non applicable; ND: not determined; Resp. assis.: requirement for respiratory assistance (invasive and non-invasive); RSV: respiratory syncytial virus; SARI: severe acute respiratory infection; y: year.

${ }^{a}$ At least one of the following: asthma, chronic cardiovascular disease, chronic respiratory disease, diabetes, exposure to tobacco, hepatic insufficiency,

immunodeficiency, neuromuscular disease, obesity, prematurity, renal insufficiency.

${ }^{b}$ Other than death.

${ }^{c}$ At least one of the following: admission in ICU, detection of pneumonia based on chest radiography, development of ARDS, resp. assis.

${ }^{d}$ In days. 
Clinical signs among SARI patients per age group, according to data from (A) all three hospitals ${ }^{\text {a }}$ participating in the study and (B) one of these three hospitals ${ }^{\mathrm{b}}$, Belgium, week 41 2018-week 22019

A. All three hospitals in the pilot SARI surveillance

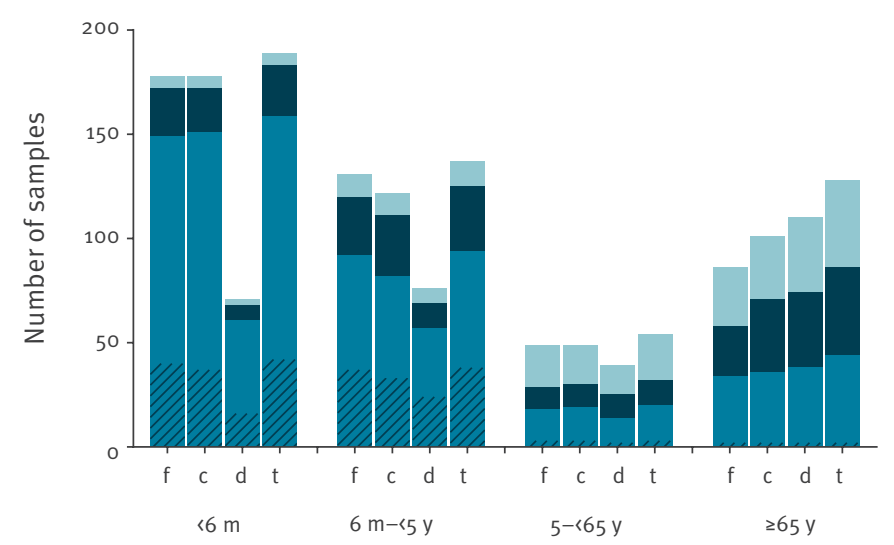

Positive for RSV (A or B)

mon Positive for both RSV and other respiratory viruses
B. One of the three hospitals in the pilot SARI surveillance

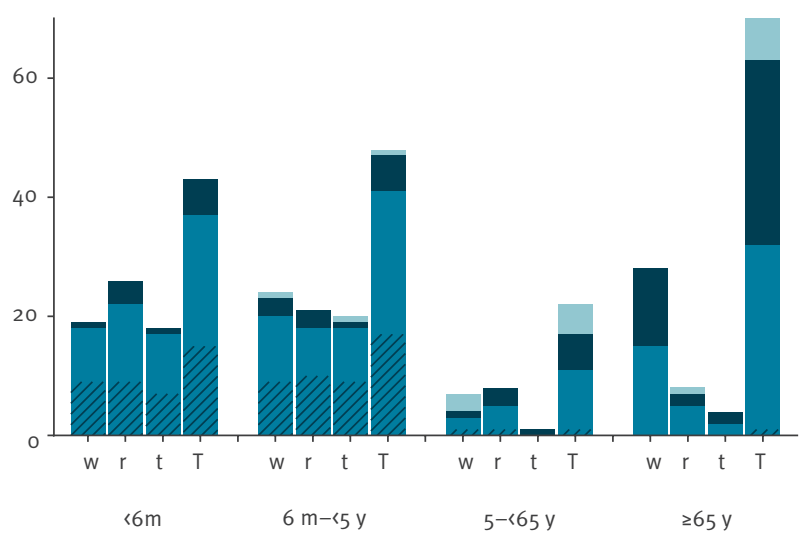

Negative for all tested respiratory viruses

Negative for RSV, positive for other respiratory viruses

C: cough; d: dyspnoea; f: fever; m: month; SARI: severe acute respiratory infection; r: rhinitis; t: tachypnoea; T: total; w: wheezing; WHO: World Health Organization; y: year.

${ }^{a}$ All three hospitals reported for each SARI case the clinical signs that were present and included in the WHO SARI case definition (cough or dyspnoea and/or fever).

b One hospital reported, for all its patients, some additional clinical signs to those in the WHO SARI case definition. These included rhinitis, tachypnoea and wheezing, as shown in panel $B$.

Age groups were defined as in the WHO's pilot study documents. In the histograms, numbers of samples per result categories, represented in different colours, are stacked (cumulative).

(52/64) for $\geq 5$ years old, Fisher exact test $p<0.001)$ (Table 2). No significant difference was observed in the proportions with fever among RSV-negative and RSV-positive patients, whatever the age group. There was some evidence of a difference in the proportion of patients with cough between the RSV-positive group $(90.9 \%, 288 / 317)$ and the RSV-negative group $(84.8 \%, 162 / 191$, Fisher exact test $p=0.044$ ) (Table 2). However, this difference was not observed when comparing within each age group. There was very good evidence that dyspnoea was more often reported for patients aged $\geq 5$ years $(81.9 \%(149 / 182))$ than among patients aged $<5$ years $(45.1 \%(147 / 326))$, Fisher exact test $p<0.001$, but without difference between RSVpositive and RSV-negative cases (Table 2 ).

In addition to these clinical signs included in the case definition and stated on the questionnaire, hospital site 1 systematically reported for all patients a detailed list of other symptoms registered by the medical staff in the patients' files. Some of these additional symptoms have been positively associated to RSV in another study [16]. Figure $4 \mathrm{~B}$ summarises the results of hospital site 1 for rhinitis, tachypnoea and wheezing. These three symptoms appeared to be more frequently reported for RSV-positive patients in the $<5$-year-olds (respectively: $48.7 \%$ (38/78), 44.9\% (35/78), 42.3\% (33/78) for RSV-positive vs 23.1\% (3/13), 23.1\% (3/13), $15.4 \%$ (2/13) for RSV-negative). In the $\geq 5$-year-olds, differences between RSV-positive and RSV-negative were not as clear (respectively: 41.9\% (18/43), 16.3\% (7/43), $4.6 \%(2 / 43)$ for RSV-positive vs $34.7 \%$ (17/49), $4.1 \%$ (2/49), 6.1\% (3/49) for RSV-negative).

\section{Risk factors and complications during hospitalisation}

Comorbidities were more common in patients aged $\geq 5$ years than in those aged $<5$ years $(92.9 \%$ $(169 / 182)$ vs $19.9 \%$ (65/326), two-sided Fisher exact test $p<0.001)$, but no difference was observed between RSV-positive and RSV-negative patients within each age group (Table 2). Supplement Table $\mathrm{S}_{1}$ details the comorbidities per age group. Among the comorbidities reported in children 16 months (24/189), the most common were premature birth (nine patients, six of whom RSV-positive), chronic respiratory diseases (five patients, all RSV-positive), tobacco exposure (four patients, three of whom RSV-positive) and chronic cardiovascular disease (one RSV-positive patient). Among the most reported comorbidities in the 6-month-to-4year-old patients $(42 / 137)$, nine were classified as premature birth (seven RSV-positive), seven as chronic 
respiratory diseases (five RSV-positive), six as asthma (four RSV-positive), six as neuromuscular diseases (five RSV-positive), five as immunodeficiencies (four RSV-positive), three as tobacco exposure (all RSVpositive) and two as chronic cardiovascular diseases (both RSV-positive). The most frequently reported comorbidities in patientsaged $\geq 5$ years were chronic respiratory diseases $(71 / 169)$, chronic cardiovascular diseases (62/169), immunodeficiency (41/169), diabetes (30/169), obesity (23/169) and asthma (19/169). Cases were relatively evenly distributed between RSVpositive, negative for all tested viruses, and only positive for another tested respiratory virus (Supplement Table S1).

There was very good evidence that the hospitalisation stay of patients aged $\geq 5$ years (median: 9 days; IQR:5-15) was longer than that of the patients aged 5 years (median:4 days; IQR:2-5) (MannWhitney $p<0.001$ ) (Table 2). Length of stay for RSVpositive cases among patient $\geq 5$ years was not different from that for the negative-for-all-testedviruses or only-positive-for-another-tested-respiratoryvirus cases (Supplement Table S1). On the contrary, in the two age groups of children aged < 5 years, hospitalisation stay was longer for RSV-positive than for RSV-negative cases. For<6-month-olds, the median stay for RSV positive patients was 4 days (IQR:3-6) vS a median of 3 days (IQR:2-4) for RSV-negative patient (Mann-Whitney $\mathrm{p}$ <0.001). For 6-month-to-4-yearolds, the median stay for RSV-positive patients was 4 days (IQR:3-5) vs a median stay of 3 days for RSVnegative patients (IQR:2-5; Mann-Whitney $\mathrm{p}=0.055$ ) (Supplement Table S1).

Twenty-two patients died during hospitalisation (four patients aged between 45 and 64 years, as well as 18 adults aged $\geq 65$ years). All four patients aged between 45 and 64 years had more than one comorbidities. Among the patients $\geq 65$ years, two had no report of comorbidity and five had only one comorbidities. By comparison, among the 35 patients aged between 45 and 64 years who survived, one had no comorbidity, 16 had one and 18 had more than one. Among the 110 surviving patients $\geq 65$ years, one had no comorbidity, 40 had one and 69 had more than one. Overall, other kinds of complications than death were more common among children aged $\geq 5$ years (97/182) than among those aged 15 years (128/326; two-sided Fisher exact test $p=0.003)$. Among the latter, some complications seemed more common among the RSV-positive patients: pneumonia (25/33), requirement for respiratory assistance (82/103), ARDS (12/15) and transfer to ICU (6/6) (Table 2).

\section{Discussion}

This pilot study showed that Belgian SARI network built to assess the severity of influenza viruses is able to capture the RSV activity if started earlier than usual. The beginning (week 41 2018) and peak (week 49 2018) of the RSV epidemic could be observed in this investigation and may have been missed by the usual SARI surveillance that started in week 22019 when the number of RSV positive samples had already considerably declined. The in-house RT-qPCRs allowed circulating RSVs to be typed and would allow to follow the change in type dominance if the current surveillance was extended to other years in the future $[17,18]$. The targeted number of patients to be included in the RSV surveillance per year [10] would have been reached if the whole network of six hospitals had taken part. This pilot study also showed that the Belgian SARI network could be used to evaluate the severity of RSV without much adaptation. The SARI network also has the advantage to operate based on a clear case definition and to allow the collection of data on several risk factors and severity indicators.

It nonetheless appears that the two main populations of interest (children, especially those aged $<6$ months, and older adults) present differences that need to be considered. Having a good case definition for the different age groups represents one of the challenges in the implementation of a surveillance system for RSV, as high sensitivity is required to avoid an underestimation of the RSV burden [19]. In this pilot study, we purposefully chose to employ the SARI case definition that has been used by the SARI network for several years, while only being less stringent for the fever criteria. This was deliberate in order to evaluate the information that could be readily obtained from our network, but this clearly represents a limitation of our study [20]. The data obtained during one season of surveillance were moreover not sufficient to perform robust measures of association between RSV and comorbidities or complications. In addition, how the comorbidities and the complications were defined and evaluated was left to each hospital to decide. Such lack of harmonisation also represents a limitation of the study that could be improved in the future. Another problem was the lack of information on the patients who did not consent to participate in the study. Of course, it would be impossible to obtain detailed information, but it could be useful to at least obtain the number of individuals lost to the surveillance and whether this should be taken into account in the analysis.

Nevertheless, the SARI case definition we used, even with a less strict fever criterion, allowed to follow RSV circulation in the children population aged 45 years and to identify comorbidities/indicators potentially associated with disease severity. Wheezing, rhinitis and tachypnoea seem like notable additional clinical signs associated with RSV-positivity in children, as already described in a previous study [16], and would need to be included in a second pilot study to fully assess their relevance in increasing the case definition sensitivity. Extending the case definition to include other symptoms such as sepsis and apnoea might also need to be considered for children 16 months, as recommended in WHO documents for RSV surveillance $[10,14,15]$. This would probably allow to better address the severity 
of disease caused by RSV in this age group, as these symptoms might be more frequently associated to severe forms [21,22]. Improving the case definition might be more challenging for older adults: the combination of clinical signs used here did not seem specific enough. Dyspnoea was more often reported in this age group, but irrespective of the RSV test result. However, a specific case definition might not be the most important point for a meaningful RSV surveillance. Assessment of the risk factors and RSV severity in this population would probably benefit more from a broader range of respiratory pathogens to be tested, including bacteria and fungi [23], in order to better evaluate the exact contribution of RSV.

These adaptations would have consequences on the actual SARI surveillance. To maintain a simple system, the case definition should not change during the whole surveillance period. The currently used SARI definition has proven effective to evaluate the severity of influenza viruses. Slightly extending it to better fit RSV would lead to an increase number of SARI cases being identified during the overall period of surveillance, which would then run from week 40 to week 20 of the following year. Since starting at week 40 would almost double the length of the surveillance, this could potentially result in almost doubling the number of collected samples. A broader case definition, with additional possible clinical signs and without fever, would lead to even more patients meeting the criteria and being enrolled. This would, however, not prevent us to identify the subset of cases responding to the more specific influenza case definition, and thus, it should not hamper us from evaluating the specific severity associated to influenza viruses. On the contrary, a broader case definition could allow the current surveillance of influenza viruses to become a surveillance system for several respiratory viruses of public health importance, such as RSV, human metapneumoviruses, and coronaviruses including the newly emerged severe acute respiratory syndrome coronavirus 2 (SARS-CoV-2) virus [3,24-27]. That being said, since the current SARI surveillance relies on the good will of the participating hospitals, the extra workload would require additional funding, as more personnel and more reagents would be required both at the hospital sites and at the national influenza centre. Given the benefits that would be gained in terms of better understanding the burden of respiratory viruses, such evolution of the SARI surveillance system might prove a more economic option on the long run.

To conclude, we showed that the implementation of a RSV surveillance based on our influenza SARI surveillance is feasible and would contribute to the objectives defined by WHO, but it would only be of interest if it can be sustained for several years. With SARI surveillance being more frequently implemented in several European countries, our study provides an example on how this surveillance can be used to gain information on RSV epidemiology.
Acknowledgements

The authors would like to thank Jeannine Weyckmans, Ilham Fdillate, Assia Hamouda and Reinout Van Eycken from the national influenza centre for their excellent technical assistance throughout the surveillance seasons; Evelyn Petit from AZ Sint-Jan, Françoise Antoine, Tiphaine Mouquet and Christelle Nguepi from CHU St-Pierre, for their dedication in the follow-up of the SARI cases; and all the doctors and nurses of the three hospitals who took part in the recruitment of the SARI cases, accepting to start the surveillance earlier.

Funding: The influenza surveillance SARI project received financial support from Belgium Federal Public Service 'Health, Food Chain Safety, and Environment', the National Insurance Health Care (INAMI/RIZIV), and the Regional Health Authorities of Flanders (AZG, Agentschap Zorg en Gezondheid) and of Wallonia (AVIQ, Agence pour une Vie de Qualité).

\section{Conflict of interest}

None declared.

\section{Authors' contributions}

Conceptualisation: CB; Data Curation: LS, CB, NB, IT; Formal Analysis: LS, CB; Investigation: $M R, M G, N D, M B, B D, C B$, IT; Resources: MR, MG, ND, MB, BD; Visualisation: LS, CB; Writing - Original Draft Preparation: LS, CB; Writing - Review \& Editing: all authors; Funding Acquisition: SQ, SVG.

\section{References}

1. Stein RT, Bont LJ, Zar H, Polack FP, Park C, Claxton A, et al. Respiratory syncytial virus hospitalization and mortality: Systematic review and meta-analysis. Pediatr Pulmonol. 2017;52(4):556-69. https://doi.org/10.1002/ppul.23570 PMID: 27740723

2. Shi T, McAllister DA, O’Brien KL, Simoes EAF, Madhi SA, Gessner BD, et al. , RSV Global Epidemiology Network. Global, regional, and national disease burden estimates of acute lower respiratory infections due to respiratory syncytial virus in young children in 2015: a systematic review and modelling study. Lancet. 2017;390(10098):946-58. https://doi. org/10.1016/S0140-6736(17)30938-8 PMID: 28689664

3. Ackerson B, Tseng HF, Sy LS, Solano Z, Slezak J, Luo Y, et al. Severe Morbidity and Mortality Associated With Respiratory Syncytial Virus Versus Influenza Infection in Hospitalized Older Adults. Clin Infect Dis. 2019;69(2):197-203. https://doi. org/10.1093/cid/ciy991 PMID: 30452608

4. Falsey AR, Hennessey PA, Formica MA, Cox C, Walsh EE. Respiratory syncytial virus infection in elderly and high-risk adults. N Engl J Med. 2005;352(17):1749-59. https://doi. org/10.1056/NEJMoa043951 PMID: 15858184

5. Shi T, Denouel A, Tietjen AK, Campbell I, Moran E, Li X, et al., RESCEU Investigators. Global Disease Burden Estimates of Respiratory Syncytial Virus-Associated Acute Respiratory Infection in Older Adults in 2015: A Systematic Review and Meta-Analysis. J Infect Dis. 2019;352(17):jizo59. https://doi. org/10.1093/infdis/jizo59 PMID: 30880339

6. European Medicines Agency. Synagis. Amsterdam: EMA. [Accessed Sep 2019]. Available from: https:// www.ema.europa.eu/en/medicines/human/EPAR/ synagis\#authorisation-details-section

7. ClinicalTrials.gov. Respiratory Syncytial Virus. [Accessed Sep 2019]. Available from: https://clinicaltrials.gov/ct2/results?con $\mathrm{d}=$ Respiratory+Syncytial+ViruS+\% $28 \mathrm{RSV} \% 29$ \&term $=\&$ cntry $=$ \& state $=\&$ city $=\&$ dist $=\&$ Search $=$ Search

8. EU Clinical Trials Register. Clinical trials for Respiratory Syncytial Virus (RSV). [Accessed Sep 2019]. Available from: https://www.clinicaltrialsregister.eu/ctr-search/search?query= Respiratory+Syncytial+Virus+(RSV)

9. Mejias A, Rodriguez-Fernandez R, Peeples ME, Ramilo 0 . Respiratory Syncytial Virus Vaccines: Are We Making 
Progress? Pediatr Infect Dis J. 2019;38(10):e266-9. https://doi. org/10.1097/INF.0000000000002404 PMID: 31335571

10. World Health Organization (WHO). WHO strategy to pilot global respiratory syncytial virus surveillance based on the Global Influenza Surveillance and Response System (口GISRS) $\square$. Geneva: WHO; 2017. Available from: https://apps.who.int/iris/ handle/10665/25985

11. Sciensano. Respiratory Syncytial Virus. 2019. Available from: https://epidemio.wiv-isp.be/ID/diseases/Pages/RSV.aspx

12. Obando-Pacheco P, Justicia-Grande AJ, Rivero-Calle I, Rodríguez-Tenreiro C, Sly P, Ramilo O, et al. Respiratory Syncytial Virus Seasonality: A Global Overview. J Infect Dis. 2018;217(9):1356-64. https://doi.org/10.1093/infdis/jiy056 PMID: 29390105

13. Broberg EK, Waris M, Johansen K, Snacken R, Penttinen $\mathrm{P}$, Network EIS, European Influenza Surveillance Network. Seasonality and geographical spread of respiratory syncytial virus epidemics in 15 European countries, 2010 to 2016. Euro Surveill. 2018;23(5):17-00284. https://doi.org/10.2807/15607917.ES.2018.23.5.17-00284 PMID: 29409569

14. Broor S, Campbell H, Hirve S, Hague S, Jackson S, Moen A, et al. Leveraging the Global Influenza Surveillance and Response System for global respiratory syncytial virus surveillanceopportunities and challenges. Influenza Other Respi Viruses. 2019;10.1111/irv.12672. https://doi.org/10.1111/irv.12672 PMID: 31444997

15. World Health Organization (WHO). WHO meeting of mid term review of the RSV surveillance pilot based on the global influenza surveillance and response system, PAHO, Washington DC, USA, 18 - 20 December 2017. Geneva: WHO; 2019. Available from: https://apps.who.int/iris/ handle/10665/311960

16. Ma X, Conrad T, Alchikh M, Reiche J, Schweiger B, Rath B. Can we distinguish respiratory viral infections based on clinical features? A prospective pediatric cohort compared to systematic literature review. Rev Med Virol. 2018;28(5):e1997. https://doi.org/10.1002/rmv.1997 PMID: 30043515

17. Vos LM, Teirlinck AC, Lozano JE, Vega T, Donker GA, Hoepelman Al, et al. Use of the moving epidemic method (MEM) to assess national surveillance data for respiratory syncytial virus (RSV) in the Netherlands, 2005 to 2017. Euro Surveill. 2019;24(20):1800469. https://doi.org/10.2807/1560-7917. ES.2019.24.20.1800469 PMID: 31115311

18. Hirsh S, Hindiyeh M, Kolet L, Regev L, Sherbany H, Yaary K, et al. Epidemiological changes of respiratory syncytial virus (RSV) infections in Israel. PLoS One. 2014;9(3):e90515. https://doi. org/10.1371/journal.pone.0090515 PMID: 24594694

19. Sáez-López E, Pechirra P, Costa I, Cristóvão P, Conde P, Machado A, et al. Performance of surveillance case definitions for respiratory syncytial virus infections through the sentinel influenza surveillance system, Portugal, 2010 to 2018. Euro Surveill. 2019;24(45):1900140. https://doi.org/10.2807/15607917.ES.2019.24.45.1900140 PMID: 31718741

20. Rha B, Dahl RM, Moyes J, Binder AM, Tempia S, Walaza S, et al. Performance of Surveillance Case Definitions in Detecting Respiratory Syncytial Virus Infection Among Young Children Hospitalized With Severe Respiratory Illness-South Africa, 2009-2014. J Pediatric Infect Dis Soc. 2019;8(4):325-33. https://doi.org/10.1093/jpids/piyo55 PMID: 29931284

21. Kneyber MCJ, Brandenburg AH, de Groot R, Joosten KFM, Rothbarth PH, Ott A, et al. Risk factors for respiratory syncytial virus associated apnoea. Eur J Pediatr. 1998;157(4):331-5. https://doi.org/10.1007/s004310050822 PMID: 9578972

22. Geoghegan S, Erviti A, Caballero MT, Vallone F, Zanone SM, Losada JV, et al. Mortality due to respiratory syncytia virus burden and risk factors. Am J Respir Crit Care Med. 2017;195(1):96-103. https://doi.org/10.1164/rccm.20160306580C PMID: 27331632

23. Jeannoël M, Lina G, Rasigade JP, Lina B, Morfin F, Casalegno JS. Microorganisms associated with respiratory syncytial virus pneumonia in the adult population. Eur ] Clin Microbiol Infect Dis. 2019;38(1):157-60. https://doi.org/10.1007/s10096-0183407-3 PMID: 30353485

24. Bordi L, Nicastri E, Scorzolini L, Di Caro A, Capobianchi MR, Castilletti C, et al. , On Behalf Of Inmi Covid-Study Group And Collaborating Centers. Differential diagnosis of illness in patients under investigation for the novel coronavirus (SARS-CoV-2), Italy, February 2020. Euro Surveill. 2020;25(8):2000170. https://doi.org/10.2807/1560-7917. ES.2020.25.8.2000170 PMID: 32127123

25. Uddin SMI, Englund JA, Kuypers JY, Chu HY, Steinhoff MC, Khatry SK, et al. Burden and risk factors for coronavirus infections in infants in rural Nepal. Clin Infect Dis. 2018;67(10):1507-14. https://doi.org/10.1093/cid/ciy317 PMID: 29668900
26. Hahn A, Wang W, Jaggi P, Dvorchik I, Ramilo O, Koranyi K, et al. Human metapneumovirus infections are associated with severe morbidity in hospitalized children of all ages. Epidemiol Infect. 2013;141(10):2213-23. https://doi.org/10.1017/ So950268812002920 PMID: 23290557

27. Li Y, Reeves RM, Wang X, Bassat Q, Brooks WA, Cohen C, et al. , RSV Global Epidemiology Network, RESCEU investigators. Global patterns in monthly activity of influenza virus, respiratory syncytial virus, parainfluenza virus, and metapneumovirus: a systematic analysis. Lancet Glob Health. 2019;7(8):e1031-45. https://doi.org/10.1016/S2214109X(19)30264-5 PMID: 31303294

\section{License, supplementary material and copyright}

This is an open-access article distributed under the terms of the Creative Commons Attribution (CC BY 4.0) Licence. You may share and adapt the material, but must give appropriate credit to the source, provide a link to the licence and indicate if changes were made.

Any supplementary material referenced in the article can be found in the online version.

This article is copyright of the authors or their affiliated institutions, 2020. 